\title{
Computational Study of Heavy Oil Production with Inflow Control Devices
}

\author{
D.I Erandi N. Wijeratne Britt M. Halvorsen \\ Department of Process, Energy and Environmental Technology, Telemark University College, \\ N-3901 Porsgrunn, Norway, \\ erandinw@yahoo.com britt.halvorsen@hit.no
}

\begin{abstract}
Recovery of heavy oil is associated with challenges in terms of early water or gas breakthrough caused by the fingering phenomenon. This study is related to Californian heavy oil production and includes 2D simulations of heavy oil in horizontal wells with autonomous inflow control devices (AICDs). Simulations have been performed to study reservoir flow, annular flow and flow through the AICDs into the production pipe using ANSYS/Fluent as the Computational Fluid Dynamics (CFD) software. A model for heavy oil production has been developed and validated against experimental data in literature. Before breakthrough the results are quite realistic which can be theoretically justified. Higher pressure drop is observed near the well bore (or across AICD) as the finger growth takes place. According to the simulated results, oil production is highly affected by fingering behaviour, since it results early water breakthrough.
\end{abstract}

Keywords: Heavy oil reservoirs, fingering phenomenon, water breakthrough, oil production, CFD, multiphase flow

\section{Introduction}

Petroleum resources are one of the major drives of the industrial development. The petroleum industry uses various technologies to increase the recovery from the existing reservoirs and enhance the well performance. The importance of heavy oil production has increased due to the decrease in the large conventional oil reservoirs. Heavy oil represents a massive world resource more than twice the size of global reserves of light or conventional oil (Safwat, 2007). There are huge, well-known resources of heavy oil, extra-heavy oil, and bitumen in Canada, Venezuela, Russia, USA and many other countries (Clark, 2007). Recovery of heavy oil is a challenging task. Formation of finger-like patterns at the water-oil interface causes early water breakthrough, which affects the oil recovery.

The production trend of heavy and light oil in California is shown in Figure 1 (Annual report of the state oil and gas supervisor, 2008).

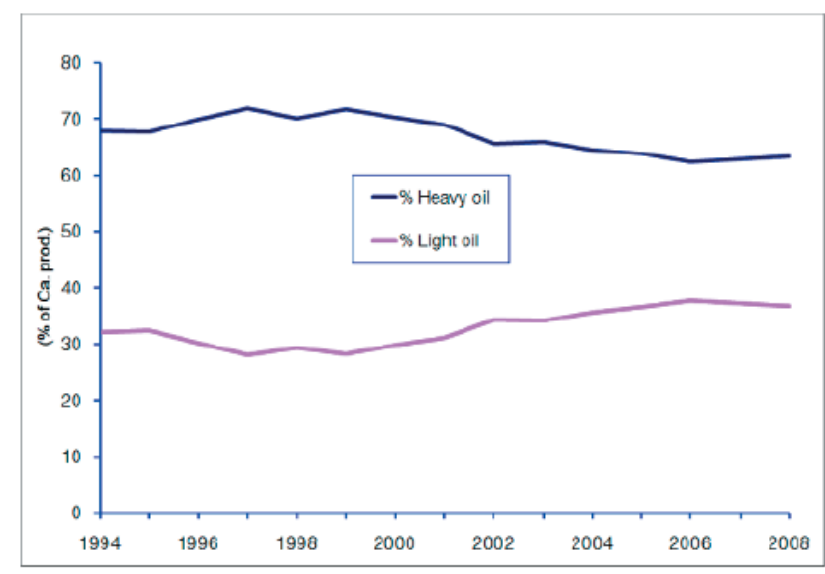

Figure 1. Heavy and light oil production in California (Annual report of the state oil and gas supervisor, 2008).

In addition to having large volumes of oil in place (OOIP), the typical California heavy oil reservoirs have favorable reservoir properties as shown in Table 1. This combination of abundant large heavy oil accumulations plus favorable reservoir properties led to California becoming the leader in U.S. heavy oil production. Challenges to the heavy oil industry include improving technology to increase the oil recovery in unusual reservoir matrices such as for example naturally fractured shales (Hanzlik, 1998).

Table 1. OOIP Weighted Median Values of California Heavy Oil Properties (Hanzlik, 1998).

\begin{tabular}{|l|l|l|}
\hline Property & Value & Unit \\
\hline Viscosity & $100-10,000$ & $\mathrm{cP}$ \\
\hline Porosity & 0.30 & - \\
\hline Permeability & 1400 & $\mathrm{mD}$ \\
\hline API Gravity & $10-20$ & 0 \\
\hline Thickness & 200 & feet \\
\hline Depth & 2500 & feet \\
\hline
\end{tabular}

A major challenge in oil production is to increase the ability to recover the oil that is in fact present in the reservoir. Estimates show that although the oil is localized and mobile, more than 50 percent of the oil is 
remaining in the reservoir after shut down. Therefore there are strong incentives for developing technologies that can contribute in increasing the oil production and recovery. In order to increase oil production and recovery it is of great importance to obtain maximum reservoir contact (MRC) and prevent the negative effects of early gas or water breakthrough. Long horizontal wells and/or multi-lateral wells are used to obtain maximum reservoir contact and the use of horizontal drilling technology in oil exploration, development and production operations has grown rapidly over past years.

Horizontal wells are used in reservoirs where early gas or water breakthrough is a problem. They are used in high permeability heavy oil reservoirs, in low permeability gas reservoirs to increase the drainage volume, in reservoirs using thermal methods for oil recovery, in reservoirs with thin oil column and in reservoirs where water flooding is used (Joshi, 1994).

The concept of Inflow Control Device (ICD) was introduced in early 90 s by Statoil researchers who observed that incremental increases in the length of horizontal wells did not create proportionately larger production rates. Further investigations revealed that this was due to the heel-toe effect (Daneshy, 2010). Different types of Inflow Control Devices are developed to delay early breakthrough by restricting the flow (Fernandes, 2009, Al-Khelaiwi, 2007). ICDs are designed to give a more uniform oil production along the horizontal well. The total oil recovery increases significantly with use of ICDs, but ICDs neither choke nor close for undesired fluids like water and gas after breakthrough has occurred.

Improvement of ICDs, Autonomous Inflow Control Devices (AICDs), are developed and the main advantage of AICDs compared to ICDs are that they choke for water and gas after breakthrough. AICDs can therefore be classified as 'smart' devices that are capable of changing their response depending on the properties of the inflowing fluid. Different types of AICDs are developed and the different principles are described in literature (Mathiesen et al., 2011; Halvorsen et al., 2012; Least et al., 2012; Aakre et al., 2013).

The objective of this paper is to make a transient computational study of fluid flow in the reservoir, fingering behavior and multiphase flow in pipe and annulus of a horizontal well with autonomous inflow control and packers. The study is related to California heavy oil reservoirs. The work focuses only on the AICD developed by Statoil ((Mathiesen et al., 2011; Halvorsen et al., 2012), and a function describing the functionality of this type of AICD is used in the simulations.

\section{Computational study of annular flow and flow through AICDs}

Computational studies of heavy oil production with water drive are performed. The simulations have been performed using ANSYS/Fluent as the Computational Fluid Dynamics (CFD) software (ANSYS FLUENT 12.0 User's Guide. 2009). Preliminary studies are performed and a model for heavy oil production has been developed and validated against experimental data (Wijerathne \& Halvorsen, 2015. Volume of Fluid (VOF) is used as the multiphase model. The VOF model can be used to model two or more immiscible fluids by solving a single set of equations and tracking the volume fraction of each of the fluids throughout the domain. The tracking of the interface(s) between the phases is accomplished by the solution of a continuity equation for the volume fraction of one (or more) of the phases. Geo-Reconstruct is used as the VOF Scheme. This is assumed to be the most accurate interface tracking scheme, and is recommended for most transient VOF calculations. The displaced fluid is as the primary phase and displacing fluid is defined as the secondary phase. The effect of surface tension along the interface between two phases is included by defining the surface tension coefficient. Porous region is specified with the viscous resistance under cell zone conditions. Since the flow rate through the porous media is very low (laminar flow), Ergun equation can be simplified as:

$$
\frac{\Delta p}{l} \approx \frac{V \mu}{\alpha}
$$

$V$ is average velocity of flow of the fluid $[\mathrm{m} / \mathrm{s}], \alpha$ is permeability of the porous medium [m2], $\mu$ is viscosity of the fluid [Pas], $\Delta p$ is pressure drop across the porous bed $[\mathrm{Pa}]$ and $l$ is thickness of the bed $[\mathrm{m}]$. The viscous resistance coefficient $(1 / \alpha)$ is defined and the inertial resistance (the turbulent component) is set to be zero. PISO (Pressure Implicit with Splitting of Operators) scheme is used as the Pressure-Velocity Coupling method, the body-force-weighted scheme as pressure discretisation scheme and second order upwind method as momentum discretization scheme.

This study covers the two-dimensional transient simulation of reservoir flow, annular flow and flow through the AICDs into the production pipe. In order to study the annular flow the geometry of the reservoir is selected according to the Figure 2. The pipe length is taken as two sections of length $12.4 \mathrm{~m}$ each. The well is completed with two AICDs in each section. The geometry, dimensions and boundary conditions are shown in Figure 2. The AICDs to the left and right side in the figure are called AICD 1 and AICD 2 respectively.

The numerical grid is drawn using GAMBIT. The AICDs and reservoir zones are named separately as two porous sections in GAMBIT and the properties of the porous sections are specified in ANSYS/Fluent. The 
created mesh is shown in Figure 3. The concentration of control volumes near the pipe is high. The sizes of the cells near the pipe and in the rest of the reservoir are $0.05 \times 0.01 \mathrm{~m} 2$ and $0.05 \times 0.05 \mathrm{~m} 2$ respectively.

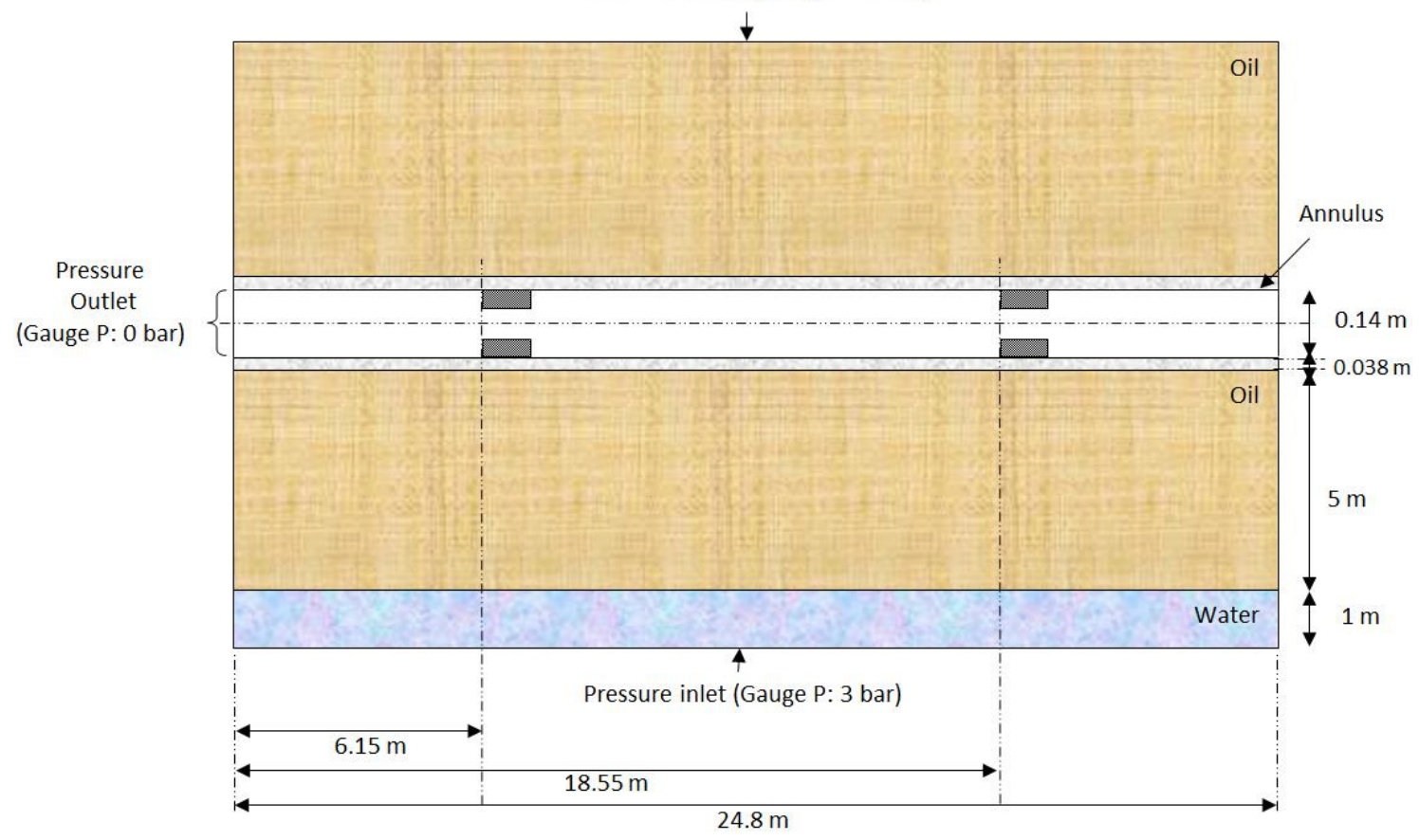

Figure 2. Geometry with the reservoir, production pipe, annulus, AICDs and boundary conditions.

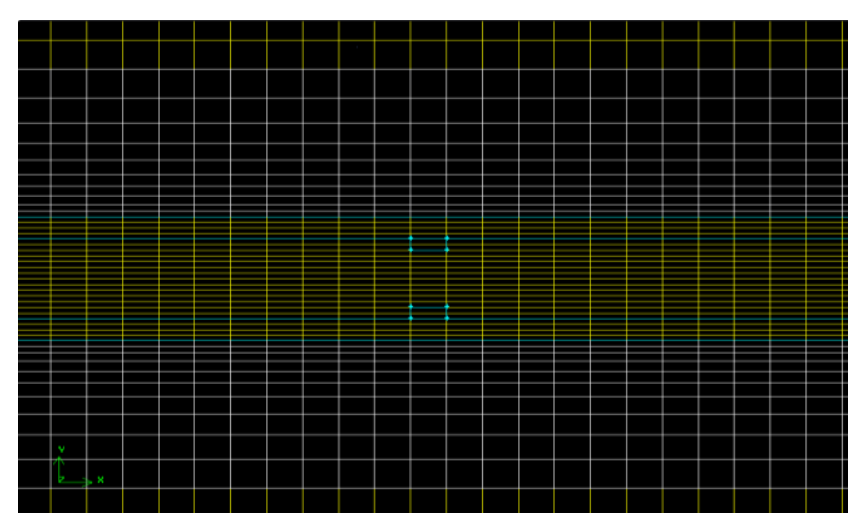

Figure 3. Enlarged view of mesh near the AICD.

\subsection{ANSYS/Fluent Model}

The reservoir properties used in the simulation are given in Table 2. The AICDs are modeled as porous regions and a power law model is describing the functionality of the valves according to Equation 2. The pressure-flow rate characteristics of the AICD is defined as below (Mathiesen et al., 2011):

$$
d P=f(\rho, \mu) \cdot a_{A I C D} \cdot q^{x}
$$

$d P$ is the differential pressure across the $\operatorname{AICD}, f(\rho, \mu)$ is an analytic function of the mixture density and viscosity, $\alpha_{A I C D}$ is a user-input 'strength' parameter, $q$ is a local volumetric mixture flow rate $\left[\mathrm{m}^{3} / \mathrm{day}\right]$ and $x$ is a user-input constant.

Table 2. Reservoir and fluid properties.

\begin{tabular}{|l|l|}
\hline Property Description & $\begin{array}{l}\text { Property } \\
\text { Value }\end{array}$ \\
\hline $\begin{array}{l}\text { Distance from the wellbore to the oil- } \\
\text { water interface [m] }\end{array}$ & 5.0 \\
\hline Water-oil interfacial tension [mN/m] & 25 \\
\hline Width of drainage area [m] & $10 \times 2$ \\
\hline Height of oil layer [m] & 10 \\
\hline Height of water layer [m] & 1.0 \\
\hline Wellbore diameter [m] & 0.22 \\
\hline Length of one horizontal section [m] & 12.4 \\
\hline Rock porosity [-] & 0.3 \\
\hline Reservoir pressure [bar] & 30 \\
\hline Well bore pressure [bar] & 27 \\
\hline Water viscosity [cP] & 0.5 \\
\hline Water density [kg/m $\left.{ }^{3}\right]$ & 1000 \\
\hline Oil viscosity [cP] & 300 \\
\hline Oil density [kg/m $\left.{ }^{3}\right]$ & 920 \\
\hline Rock permeability [D] & 3.0 \\
\hline
\end{tabular}


The function $f(\rho, \mu)$ is presented in Equation 3.

$$
f(\rho, \mu)=\left[\frac{\rho_{m i x}^{2}}{\rho_{c a l}}\right] \cdot\left[\frac{\mu_{c a l}}{\mu_{m i x}}\right]^{y}
$$

$\rho_{c a l}$ and $\mu_{c a l}$ are the calibration density and viscosity, properties of water typically, and $y$ is a user-input constant. This implies that the pressure drop across AICD depends on a combination of the fluid properties and design variables. Constants used for the AICD valve function in this study are tuned based on data presented by Statoil (Mathiesen, 2011; Halvorsen, 2012) and are not exact. In the ANSYS Porous model (Flow Through Porous Media, 2007), the differential pressure per length is expressed as:

$$
\frac{d P}{l}=C_{0}|v|^{C_{1}}
$$

Based on the tuned differential pressure calculated from Equation 2, the constants $C_{0}$ and $C_{l}$ for oil water and gas are estimated and used in the simulations. Figure 4 shows the theoretical performance curves for oil water and gas through the AICD. The curves show that the flow rate as a function of drawdown is much higher for viscous oil $(300 \mathrm{cP})$ than for water and gas. This shows that the AICD is capable of choking for water and gas. The boundary conditions are set as shown in Figure 2 . The numerical values of the boundary conditions are presented in Table 2.

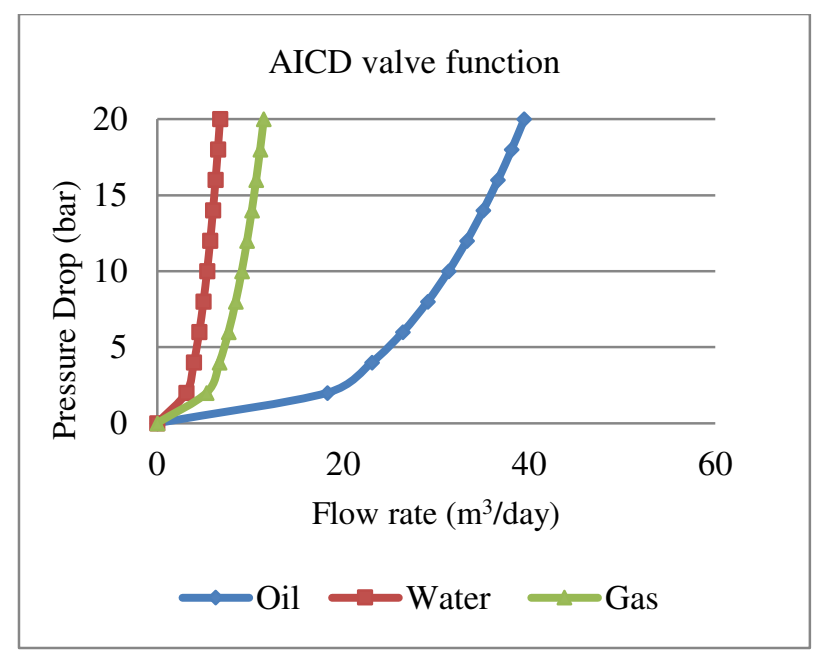

Figure 4. AICD valve function for oil, water and gas flow.

\section{Results and Discussion}

The velocity of the water/oil contact (WOC) just before any instability occurs is shown in Figure 5. The average velocity of the WOC is $7.07 \mathrm{e}-7 \mathrm{~m} / \mathrm{s}$. The critical velocity $\mathrm{U}_{\mathrm{C}}$ above which instabilities occur is calculated from:

$$
U_{c}=\left(\rho_{1}-\rho_{2}\right) g K /\left(\mu_{1}-\mu_{2}\right)
$$

$K$ is the permeability, $\rho$ the density and $\mu$ the viscosity. The critical velocity, $U_{C}$ is calculated based on the fluid properties and the permeability presented in Table 2 . The critical velocity is $7.75 \mathrm{e}-9 \mathrm{~m} / \mathrm{s}$ (upwards). The average WOC velocity is higher than the critical velocity above which instabilities occurs, and this states that finger formation in the reservoir can be expected. Figure 6 shows the phase distribution, static pressure distribution and velocity vectors near the AICDs after short time of production, before the breakthrough. Initial fingering can be observed (a), the pressure decreases gradually towards the well (b) and the velocity profile has the direction towards the AICD in the annulus, and a typical laminar velocity profile in the pipeline (c). Figure 7 shows the pressure profile along the $\mathrm{Y}$ axis (vertical direction) through AICD 1 and 2 before breakthrough. The two curves have identical shape.

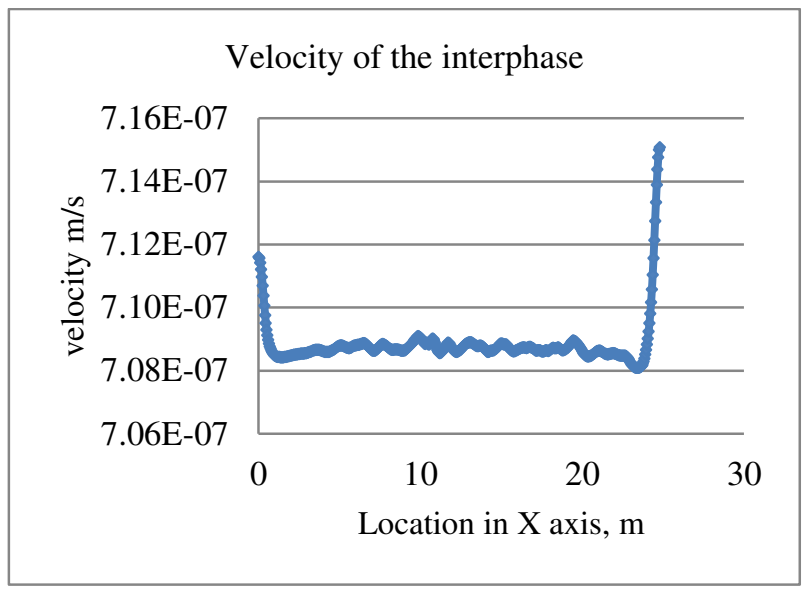

Figure 5. Velocity of WOC before fingers are formed

Figure 8a shows the contours of oil volume fraction at the time of breakthrough to the annular region and Figure $8 \mathrm{~b}$ shows a closer view near the breakthrough location. Breakthrough to the annular region occurs after about 10.4 days. The effective interface is parallel to the production pipe, due to the negligible pressure drop in the short pipe. The long fingers at the two ends may not reflect the actual scenario, since these fingers are formed due to the high velocities observed in Figure 5.

There are few dominant fingers which have outstripped the adjacent fingers. They have mushroomed as a result of the lateral pressure gradients existing near the end of these fingers. The subsequent development of this mushrooming continues until the break- through to the annular space takes place. Between the water fingers, areas of residual oil are left without being influenced by the water flooding.

The resultant fingering pattern of the simulation shows the tendency of disintegrating the viscous fingers into bubbles. Some experimental data from literature showed that the tips of the fingers are spreading while the tails of the fingers are narrowing. The less viscous fluid has high velocities in these narrowing regions, which can ultimately cause the formation of a separated island of the less viscous fluid. This can also be due to interface tension 
forces which try to minimize the surface area to achieve stability.

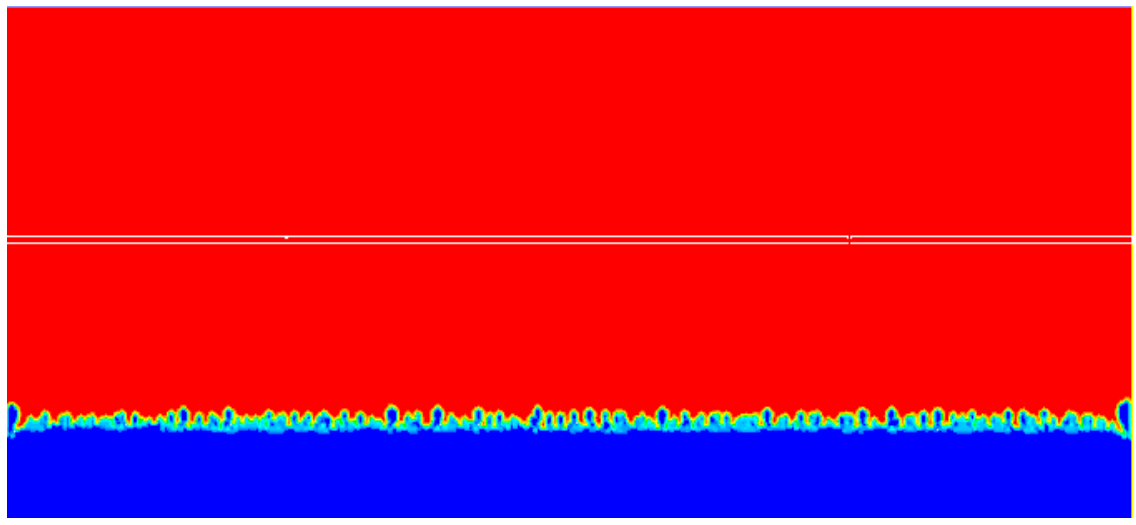

Figure 6(a). Phase distribution after short time of production. Blue indicates water and red indicates oil.

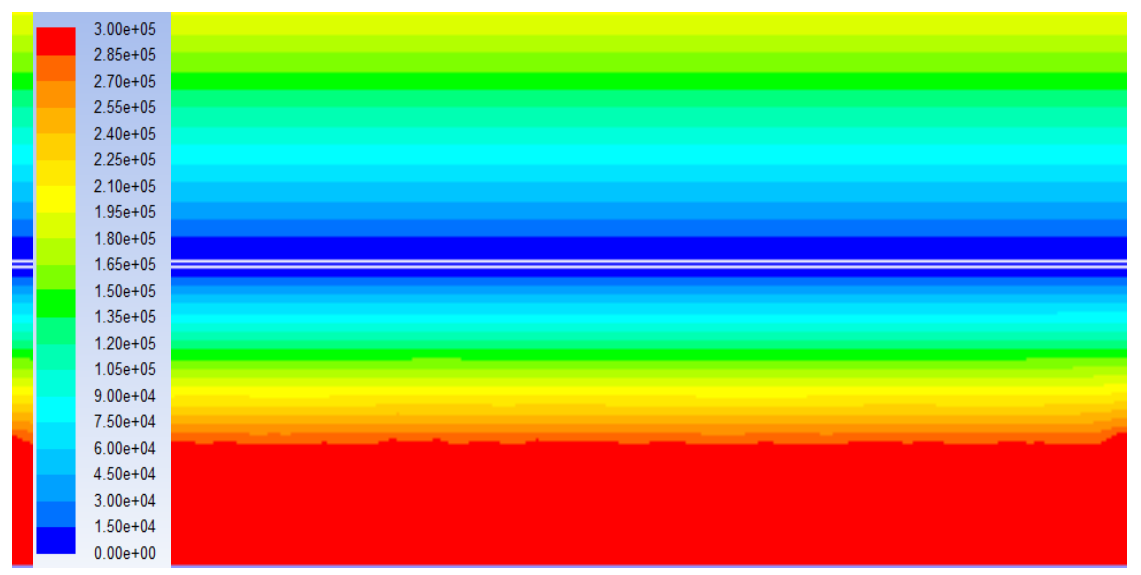

Figure 6(b). Static Pressure distribution after short time of production. Red is 3 bar gauge pressure and blue is 0 bar gauge pressure related to the well.

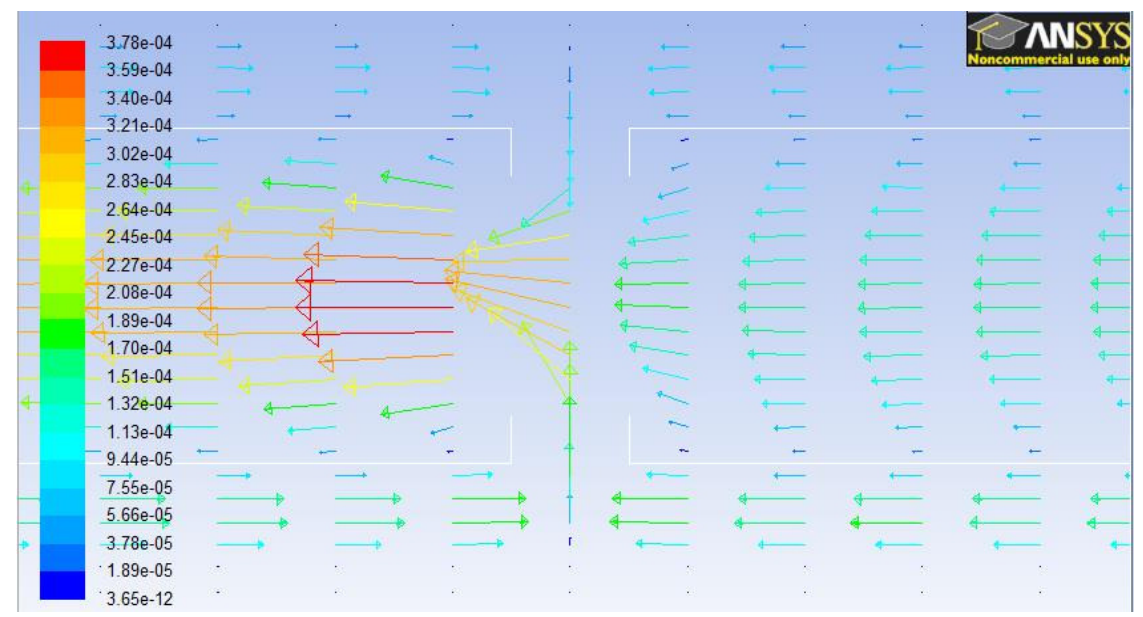

Figure 6(c) Velocity vectors near AICD after short time of production. The red arrows indicates higher velocity and the blue lower velocity. Range: $3.66 \cdot 10^{-12}$ to $3.76 \cdot 10^{-4} \mathrm{~m} / \mathrm{s}$. 


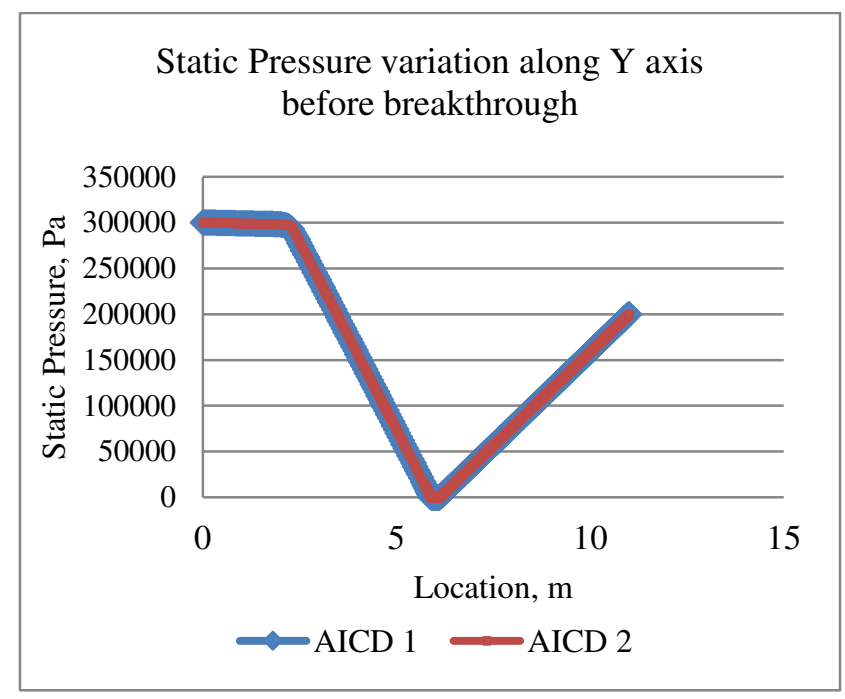

Figure 7. Static Pressure variation along the y axis through AICD 1 and 2 after short time of production (before breakthrough).

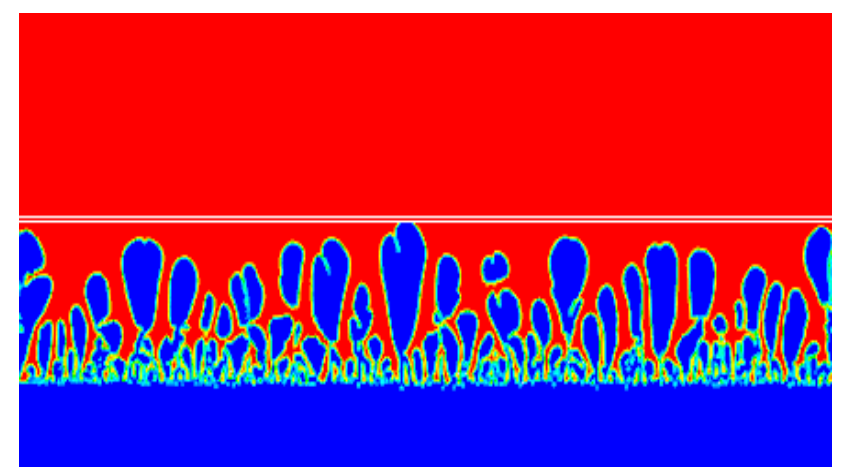

Figure 8(a). Phase distribution at the breakthrough. Blue and red represents water and oil respectively.

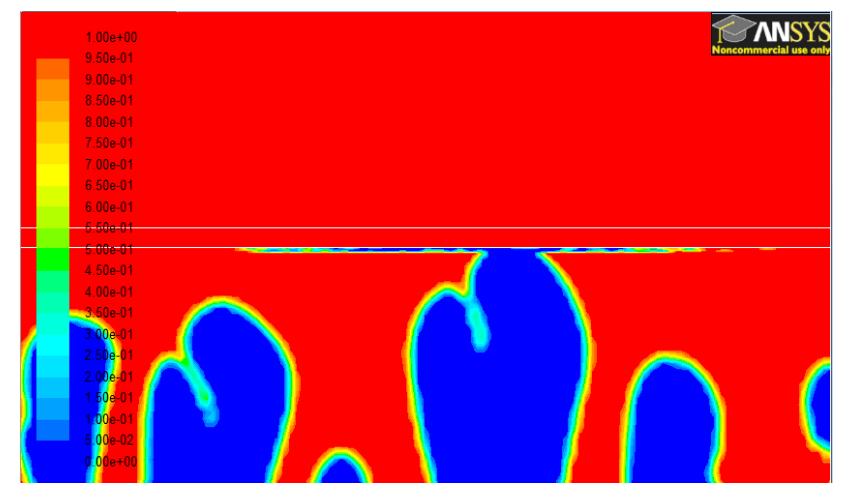

Figure 8(b). Closer view of finger at the breakthrough.

An additional simulation with a very fine numerical grid was carried out on a smaller part of the reservoir in order to check the grid size dependency on the fingering pattern. The resultant fingering pattern is shown in Figure 9. The fingers have better clear cut edges than the fingers observed in Figure 8. The fingers do not seem to disintegrate into bubbles, but narrowing points in the tail of the fingers are observed. The fingering pattern can be more complex under real reservoir conditions due to heterogeneities of the rock, wetting and non-wetting characteristics of oil and water, initial and residual saturation conditions, the open pore volume available for flow and depletion of initial

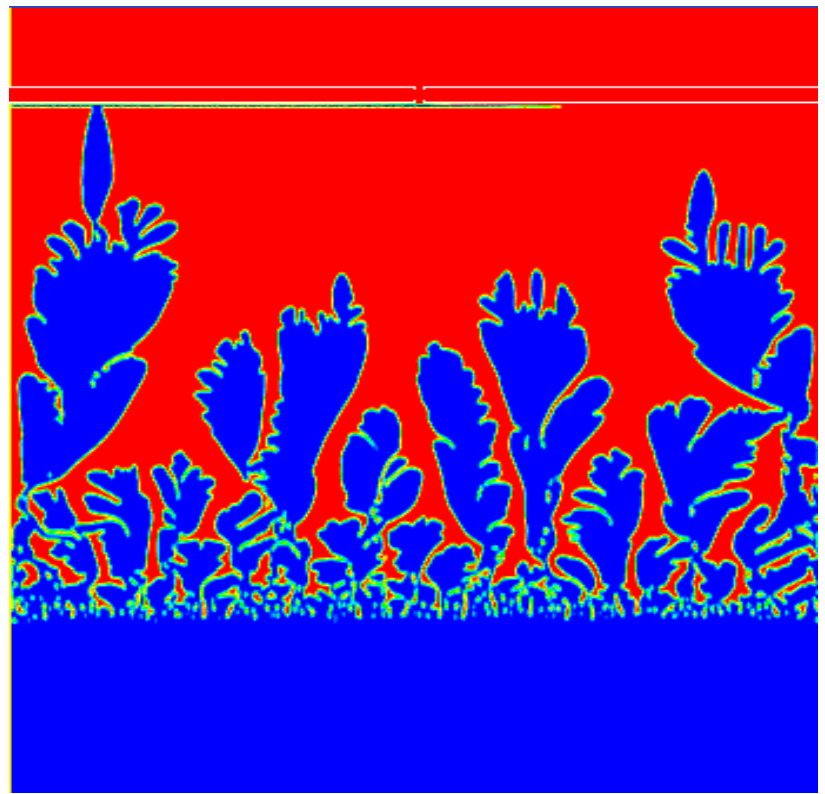

Figure 9. Fingering pattern caused by a very fine grid. Blue and red represents water and oil respectively.

reservoir conditions as production proceeds.

Figure 10 shows the contours of static pressure at the breakthrough. As the fingers develop towards the production pipe, the inlet pressure (colored in red) also develops in the same direction. At breakthrough, the pressure in the finger becomes approximately same as the pressure of the annular region.

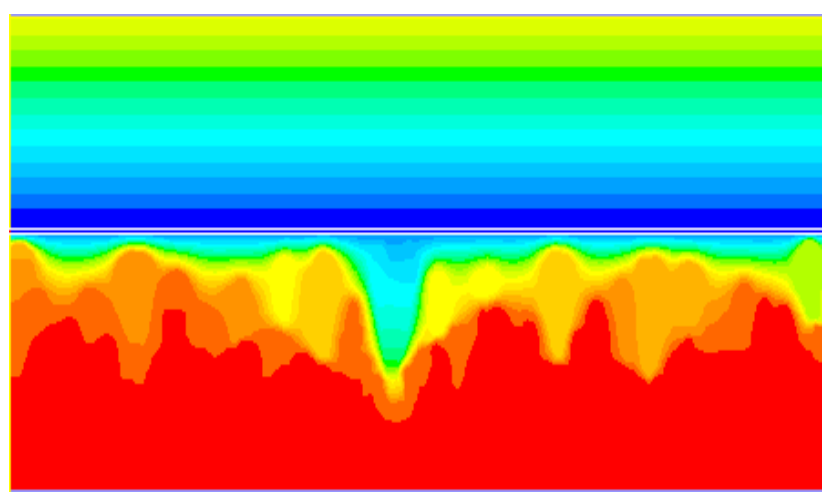

Figure 10. Static pressure distribution at the breakthrough. Pressure gauge range from 0bar (blue) to 3 bar (red).

Figure 11 shows the static pressure variation as a function of vertical position in the reservoir at breakthrough. It is clearly seen that the pressure gradient is very steep near the well bore due to the low pressure drop of water fingering through the reservoir. Comparing Figure 7 (static pressure before after short time of production) with the static pressure at breakthrough, it can be seen that the static pressure profile has changed significantly. This indicates that large 
amounts of water can be produced in the breakthrough zone if inflow control is not used to restrict the flow. The change in static pressure profile only occurs below the well where the water layer approaches the pipe.

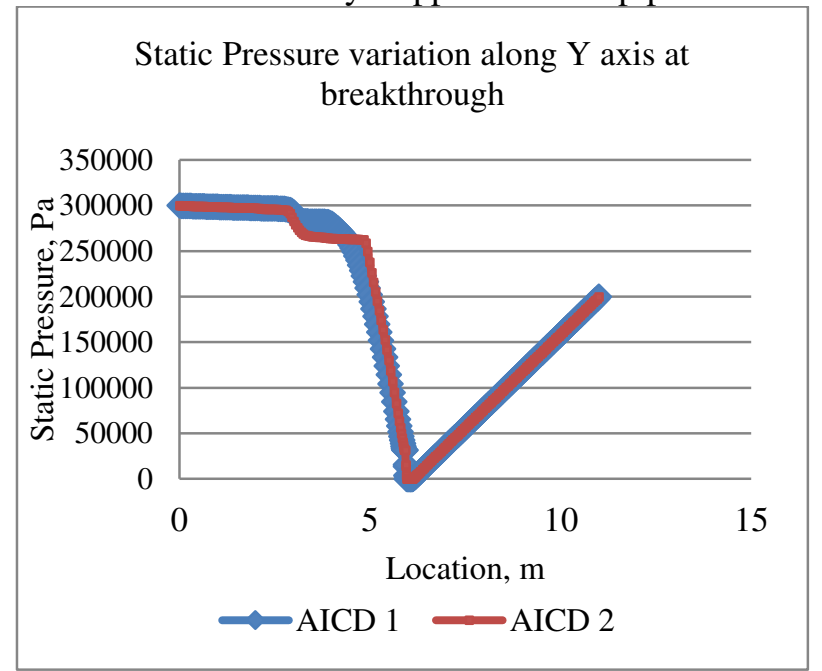

Figure 11. Static Pressure variation along $\mathrm{Y}$ axis through AICD 1 and 2 at breakthrough.

After the breakthrough, the simulation was run with maximum time step size 3-4 seconds and with Courant Number equals 70 . Breakthrough to the pipe occurs after 10.5 days of production. Breakthrough occurs through the AICD 1 (water side). There is no flow restriction in the annular region compared to through the porous media. Figure 12 shows the breakthrough to the pipe via AICD 1. The white circles show the location of AICDs. The breakthrough occurs after short time through AICD 2 as well due to the unrestricted flow through the annulus.
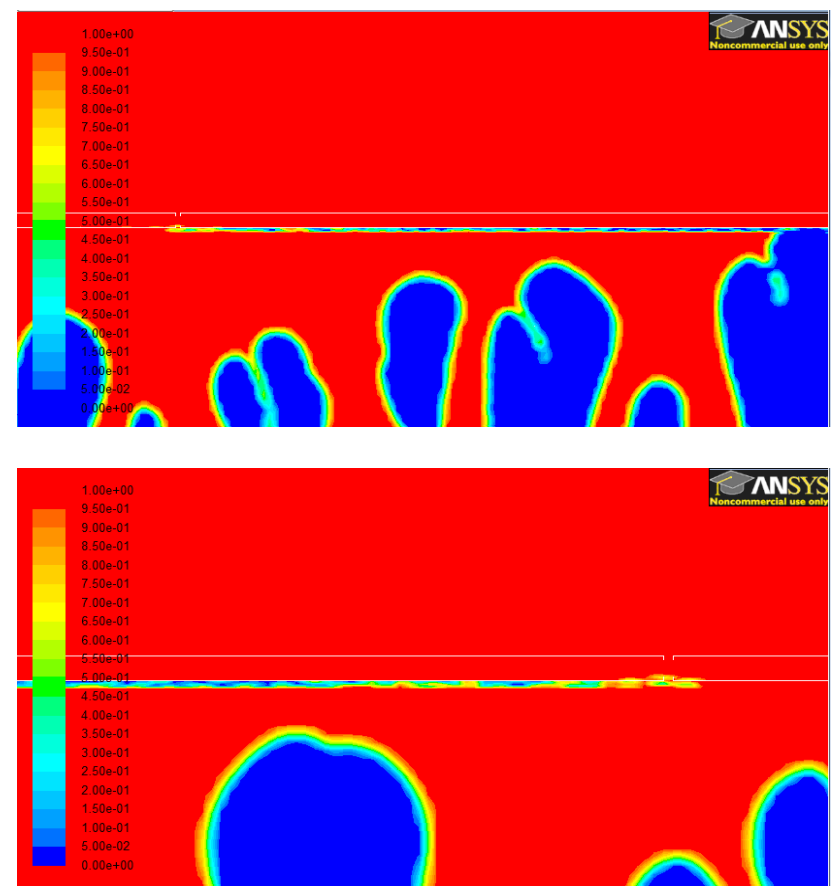

Figure 12. Breakthrough to the pipe via AICD1 (upper) and AICD2 (lower).
According to Figure 12, it is clear that packers in the annulus between the AICDs can delay the breakthrough through AICD 2. Figure 13 shows the volumetric flow rate of oil and water at the outlet of the well.

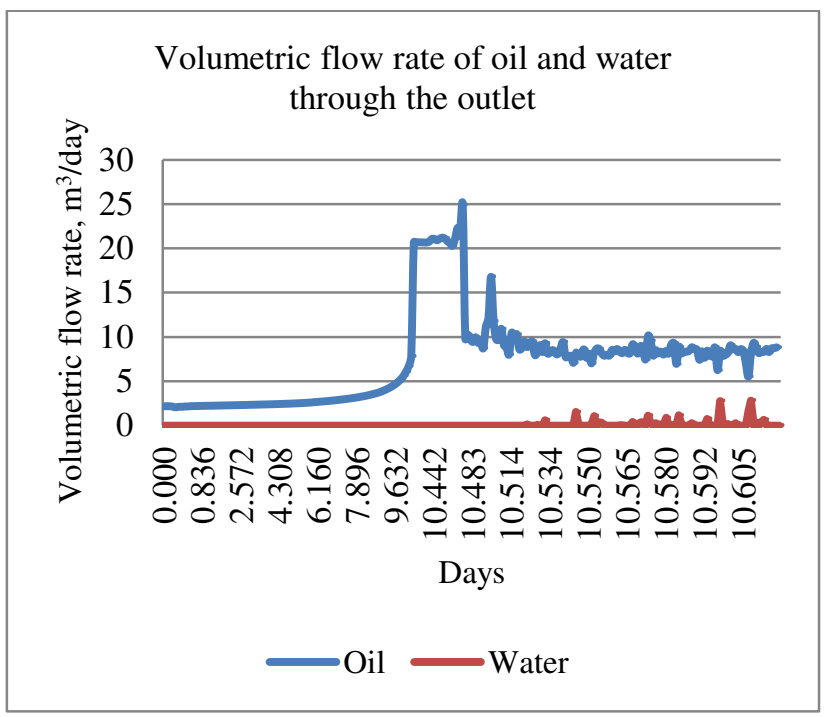

Figure 13. Volumetric flow rate of oil and water through the outlet.

The oil flow rate increases significantly before breakthrough, and decreases again after breakthrough occurs and the AICDs choke the flow into the well. The irregular peaks in the water flow rate imply that water has started to flow. After the breakthrough, the oil flow rate is still higher than before breakthrough. This is due to the increase drawdown and the high flow rate of oil into the well in a period before the breakthrough. Due to two-dimensional simulations, the AICDs in the upper wall of the pipeline are not influenced by the water flow in the annulus, and are still producing oil without any restrictions. Both oil and water flow rates are expected to decrease slowly after some time until oil is again surrounding the AICDs.

The two-dimensional computational study has shown that the fingering phenomenon occurs in heavy oil reservoirs with water drive. The simulations have also predicted the early breakthrough of water to the annulus and the well and the functionality of the AICD. The AICDs restricted the flow at water breakthrough, which indicated that the modelling of the AICD in Fluent agreed well with the expected functionality of the AICD. However, to investigate the potential of increased oil recovery with AICD, a much longer well with several AICDs and packers to isolate the breakthrough zones has to be simulated. The total picture of the production of heavy oil with water drive can only be obtained by using three-dimensional simulation. 


\section{Conclusion}

Recovery of heavy oil is associated with difficulties in terms of early water or gas breakthrough caused by the fingering phenomenon. This study is related to Californian heavy oil production and includes 2D simulations of heavy oil with viscosity $300 \mathrm{cP}$ in horizontal wells with autonomous inflow control devices (AICDs). The simulations have been performed to study reservoir flow, annular flow and flow through the AICDs into the production pipe using ANSYS/Fluent as the Computational Fluid Dynamics (CFD) software. Volume of Fluid (VOF) is used as the multiphase model. Preliminary studies are performed and a model for heavy oil production has been developed and validated against experimental data found in literature.

Before the breakthrough, the analysis of data gives quite realistic results which can be theoretically justified. The simulations clearly stated the fingering phenomenon and the effect on breakthrough and oil production. The simulation time after the breakthrough is very short due to multiphase flow in pipeline, which leads to instabilities. Higher pressure drop is observed near the well bore (or across AICD) as the finger growth takes place. Simulation of two pipe sections in a homogeneous reservoir does not reflect the function of AICDs adequately. This is due to negligible frictional pressure drop in the pipe which breakthrough at approximately the same time in the two sections. This study has detected/identified/proven the formation of fingering in heavy oil reservoir with water drive and according to the simulated results, oil production is highly affected by fingering behavior, since it enables the early water breakthrough while most of the oil is left unproduced. However, further simulations are needed to study the effect of inflow control devices. Further simulations should also involve packers to isolate the breakthrough zones.

\section{References}

H. Aakre, B. Halvorsen, B. Werswick, V. Mathiesen, Smart well with autonomous inflow control valve technology, in: SPE Middle East Oil and Gas Show and Exhibition, 2013.

F. Al-Khelaiwi, D. Davies, Inflow control devices: Application and value quantification of a developing technology, in: International Oil Conference and Exhibtion, 2007.

B. Clark, Heavy oil, extra-heavy oil and bitumen, Tech. rep., National Petroleum Council (2007).

C. D. of Conservation, Annual report of the state oil and gas supervisor, Tech. rep., California Department of Conservation, Division of Oil, Gas and Geothermal Resources (2008).

A. Daneshy, Icd design: Revisiting objectives and techniques, in: SPE Asia Pacific Oil and Gas Conference and Exhibition, 2010.

P. Fernandes, Z. Li, D. Zhu, Understanding the roles of inflow-control devices in optimizing horizontalwell performance, in: SPE Annual Technical Conference and Exhibition, 2009.
M. Halvorsen, O. Naevdal, G. Elseth, Increased oil production by autonomous inflow control with rcp valves, in: SPE Annual Technical Conference and Exhibition, 2012

E. Hanzlik, Status of heavy oil and tar sand resources in the United States, Tech. rep., UNITAR Center for Heavy Crude and Tar Sands (1998)

Incorporation, F., ANSYS FLUENT 12.0 User's Guide, 2009.

Incorporation, F., Flow Through Porous Media. 2007.

S. Joshi, Horizontal wells: Successes and failures, Journal of Canadian Petroleum Technology 33(1994).

B. Least, S. Greci, R. Burkey, A. Utford, A. Wilema, Autonomous icd single phase testing, in: SPE Annual Technical Conference and Exhibition, 2012.

V. Mathiesen, H. Aakre, B. Werswick, G. Elseth, The autonomous rcp valve - new technology for inflow control in horizontal wells, in: SPE Annual Technical Conference and Exhibition, 2011.

D. Mud, Horizontal well development of agravity drainage reservoir, in: SPE Western Regional Meeting, 1996.

M. Safwat, Challenges of heavy oil production, OAPEC - IFP Seminar, 2007.

D.I.Erandi N. Wijerathne, Britt M. Halvorsen, Computational study of fingering phenomenon in heavy oil reservoir with water drive, Fuel 158, 2015. 